Pacific Journal of Mathematics

IRREDUCIBLE SUBGROUPS OF ORTHOGONAL GROUPS 


\title{
IRREDUCIBLE SUBGROUPS OF ORTHOGONAL GROUPS GENERATED BY GROUPS OF ROOT TYPE I
}

\author{
BetTy SAlzberg Stark
}

\begin{abstract}
In this paper it is shown that the only irreducible subgroups of orthogonal groups over finite fields of odd characteristic generated by groups of root type 1 are (1) unitary groups, (2) groups of type $G_{2}$, or (3) the commutator subgroups of the orthogonal groups in question. This is a generalization of a previous result by the author.
\end{abstract}

In a previous paper [6], the author proved that the only subgroups of orthogonal groups over finite fields of odd characteristic which are generated by groups of root type 1 (defined below) and transitive on one-dimensional singular subspaces are the groups (1), (2), and (3) above.

The result of this paper is related to two current lines of research. Firstly we note that the irreducible groups that we study, together with the vector spaces they act on, form a quadratic pair in the sense of Thompson [8] [see e.g., 4], since the elements of a group of root type 1 have a minimal polynomial $(x-1)^{2}$.

Thus we have a classification of certain subgroups of orthogonal groups which form a quadratic pair. However, our methods depend on the orthogonal geometry and hence are unlike the methods of Thompson. In addition, we include the case $p=3$, not considered by Thompson.

Secondly, the groups of root type 1 form a conjugacy class in the orthogonal groups as well as in the groups (1), (2), and (3) above. (This is not proved here, but is proved in [8] $\S 16$, where Thompson shows $\Sigma$ is a conjugacy class in $G$, and can also be proved directly.) Furthermore, any two groups of root type 1 generate either an abelian $p$-group or $\mathrm{SL}(2, q)$ (where $q$ is the number of elements in a group of root type 1) or a group of order $q^{3}$ which is isomorphic to the Sylow $p$-subgroup of SL $(3, q)$. Thus our result is analogous to results obtained by, for example, Aschbacker [2], or Fischer [3], who have investigated the classification of groups generated by a conjugacy class of $p$-elements such that any two $p$-elements generate a group from a given class of groups.

1. Terminology and restatement of theorem. Let $V$ be a finite dimensional vector space over a finite field of characteristic not 2 . Let $B$ be a symmetric bilinear form on $V$. $B$ determines a quadratic 
form $Q$ on $V$ by $B(x, x)=2 Q(x)$. In addition, suppose there is no $x \neq 0$ in $V$ such that $B(x, y)=0$ for all $y$ in $V$. Then we say $B$ is nondegenerate on $V$. In this case, the group of linear transformations on $V$ preserving $B$ is called the orthogonal group (with respect to $B$ ), and is denoted $0(V)$. The commutator subgroup of $0(V)$ is denoted $\Omega(V)$.

If for all $x \in S \neq\{0\}, S \subseteq V, B(x, x)=0$, we say $S$ is singular. We remark that the condition $S \neq\{0\}$ is not standard. Our "singular vectors" and "singular subspaces" are always nonzero. If a vector $u$ is nonzero and not singular, it is nonsingular. This is standard. Further, we use projective terminology. Thus, a one-dimensional subspace is a point and a two-dimensional subspace is a line. Let \langle\rangle denote "subspace generated by". Thus $\langle x\rangle$ is the point generated by the vector $x$.

The set of vectors $x$ such that $B(x, y)=0$ for all $y \in Y \subseteq V$ is denoted $Y^{\perp}$. If $X \subseteq Y^{\llcorner}$we say $X$ is perpendicular to $Y$. Since $B$ is bilinear, this is equivalent to saying $\langle X\rangle$ is perpendicular to $\langle Y\rangle$. Since $B$ is symmetric, $X \subseteq Y^{-}$implies $Y \subseteq X^{\perp}$.

Now let $x$ be a singular vector (hence nonzero by definition), and let $u$ be in $x^{-}$. Define a linear transformation $\rho_{x, u}$ by: For $z \in x^{-}$, $\rho_{x, u}$ sends $z$ to $z+B(z, u) x$. This transformation preserves $B$ on the $n-1$ dimensional space $x^{-}$. (Note that in case $u \in\langle x\rangle \rho_{x, u}$ acts as the identity on $x^{\perp}$.) By Witt's theorem (see, for example, Artin [1, p. 121]), every linear transformation which preserves $B$ on a subspace of $V$ can be extended to a member of $0(V)$. Tamagawa [7] shows that the extension $\rho$ of $\rho_{x, u}$ to a member of $0(V)$ is unique. In fact, if $y$ is a singular vector such that $B(y, u)=0$ and $B(x, y)=1$ (when $u \in\langle x\rangle$, such vectors always exist) then $\rho$ sends $y$ to $y-Q(u) x-u$. We abuse notation by allowing $\rho_{x, u}$ to stand for its extension to a member of $0(V) ; \rho_{x, u}$ is called a Siegel transformation.

Since, as a direct consequence of its definition,

$$
\rho_{x, u} \rho_{x, v}=\rho_{x, u+v}
$$

we see that the set $\Sigma=\left\{\rho_{x, k u} \mid x\right.$ singular, $u \in x^{+}, u \notin\langle x\rangle ; x, u$ fixed, $k \in F\}$ is a group isomorphic to the additive group of $F$. If $u$ is singular we say $\Sigma$ is a group of root type 1 . If $u$ is nongingular we say $\Sigma$ is a group of root type 2 .

Let $|F|=q=p^{n}$, where $p$ is an odd prime. Let $\operatorname{dim} V=t$ and index $V=\nu$. We remark that if $\Omega(V)$ contains a group of root type $1, \nu$ must be at last 2 , and hence $t$ is at least 4 . We restate the main theorem of this paper:

MaIN Theorem. Let $G$ be an irreducible subgroup of $Q(V)$ generated by groups of root type 1 . Then 
(i) $G \cong G_{2}(q)$

(ii) $G \cong S U\left(t / 2, q^{2}\right)$ and either

$$
t=4 m+2, \nu=2 m, m \geqq 1 \text { or } t=4 m, \nu=2 m, m>1
$$

or

(iii) $G=\Omega(V)$.

2. The use of standard basis notation. Artin [1] shows that $V$ can always be represented in the following way:

$$
V=\left\langle x_{1}, x_{-1}\right\rangle \perp\left\langle x_{2}, x_{-2}\right\rangle \perp \cdots\left\langle x_{k}, x_{-k}\right\rangle \perp w
$$

where $\left\langle x_{i}, x_{-i}\right\rangle$ is a "hyperbolic line",

i.e.,

$$
B\left(x_{i}, x_{-i}\right)=1 \text { and } Q\left(x_{i}\right)=Q\left(x_{-i}\right)=0
$$

and $W$ is anisotropic (containing no singular points). The dimension of $W$ is 1,2 or 0 . The index of $V$ is $k$.

By Witt's theorem, if $v_{1}, \cdots, v_{t}$ are any vectors in $V$ with the same multiplication table $B\left(v_{i}, v_{j}\right)$ as some subset of a standard basis, they can be extended to a standard basis. Thus we use $\left\langle x_{1}, x_{2}\right\rangle$, for example, to stand for any singular line in $V$.

3. Preliminary results. We restate for the reader some of the results from [6].

LEMMA 1. The groups of root type 1 are in one-to-one correspondence with the singular lines of $V$.

The follows from elementary properties of Siegel transformations, namely

$$
\begin{array}{rl}
\rho_{x, u+k x}=\rho_{x, u} & k \in F \\
\rho_{x, c u}=\rho_{c x, u} & c \in F^{*}
\end{array}
$$

and for singular $u$

$$
\rho_{x, u}=\rho_{-u, x} .
$$

Thus we may make the following definition:

Let $\Sigma l=\left\{\rho_{x, u} \mid l=\langle x, u\rangle\right\} \cup\{1\}$.

If $\Sigma l \subseteq G$, say $l$ is an axis line for $G$.

For the following lemmas let $\Sigma l$ and $\Sigma m$ be groups of root type 1 and let $H$ be the group generated by $\Sigma l$ and $\Sigma m$. Let $W=\langle l, m\rangle$ and suppose $l \cap m=\{0\}$. 
LEMMA 2.

(1) If $l \cap m^{\perp}=l, \quad H$ is abelian.

(2) If $l \cap m^{\perp}=P$, (a point),

$H$ is isomorphic to the p-Sylow subgroup of $\mathrm{SL}_{3}(q)\left(q=p^{n}\right)$.

(3) If $l \cap m^{\perp}=\{0\}, H \cong \mathrm{SL}_{2}(q)$.

Lemma 3. If $l \cap m^{\llcorner}=\{0\}, H$ is reducible on $W$, every singular point in $W$ is on exactly one axis line for $H$, and the orbits of singular vectors under $H$ form singular lines.

To illustrate, if $l=\left\langle x_{1}, x_{2}\right\rangle, m=\left\langle x_{-1}, x_{-2}\right\rangle$, then $\left\langle x_{1}, x_{-2}\right\rangle$ is an orbit of singular vectors under $H$, i.e., for any $c, d \in F$, not both zero there is a $\sigma \in H$ such that $\sigma\left(x_{-2}\right)=c x_{1}+d x_{-2}$.

Lemma 4. If $l \cap m^{\perp}=P, P$ is on two distinct axis lines in $H$.

To illustrate Lemma 4, let $l=\left\langle x_{1}, x_{2}\right\rangle m=\left\langle x_{-1}, x_{3}\right\rangle$. Then $P=\left\langle x_{2}\right\rangle$, and $\left\langle x_{2}, x_{3}\right\rangle$ as well as $\left\langle x_{2}, x_{1}\right\rangle$ are axis lines for $H$.

Lemma 5. If $l \cap m^{\perp}=\{0\}, H$ is transitive on the nonsingular vectors of a given length in $W$.

4. Baer's theorem. In Lemma 2 we have remarked that two groups of root type 1 either generate a $p$ group or $\mathrm{SL}_{2}(q)$. In the latter case, we say the two groups of root type 1 are opposites, following Thompson [8]. Baer's theorem allows us to assume that each group of root type 1 in an irreducible subgroup of $\Omega(V)$ has an opposite. For the convenience of the reader we state the relevant theorems:

Lemma 6 (Baer). (Theorem 3.8.2 [5]). Let $K$ be a conjugacy class of p-elements of the group $G$. If every pair of elements of $K$ generate a p-group then $K$ lies in a normal p-subgroup of $G$.

LEMMA 7 (Theorem 3.1.3 [5]). If $G$ possesses a faithful irreducible representation on a vector space over a field of characteristic $p$, then $G$ has no nontrivial normal p-subgroups.

5. No two axis lines intersect (Case 1 ). In $\S \S 5$ and 6 we assume that no two axis lines of $G$ intersect. Suppose $l=\left\langle x_{1}, x_{2}\right\rangle$ is an axis line for $G$. By Baer's theorem (Lemmas 6 and 7), we must have an opposite, " $m$ ". Without loss of generality, we may use standard basis notation and let $m=\left\langle x_{-1}, x_{-2}\right\rangle$. Each singular point in $W=\langle l, m\rangle$ is on exactly one axis line for $H$, the group generated by $\Sigma l$ and $\Sigma m$. 
We retain these definitions for $H, W, l$, and $m$ in $\S \S 5$ and 6 . Thus if $G$ is to be irreducible and have no two axis lines intersect, the dimension of $V$ must be greater than 4 .

In order that $\left\langle x_{1}, x_{-2}\right\rangle=X$ not be fixed by $G$, some point in $X$ must be moved by an element of $G$ out of $X$. Without loss of generality (by Lemma 3) let us assume $\left\langle x_{1}\right\rangle$ is moved out of $X$. Then we must have an axis line of form $n=\left\langle x_{-1}+y, z\right\rangle$. We may assume $z \in x_{1}^{\perp}$ since $n \cap x_{1}^{\perp}$ has dimension one. If $z \in\left\langle x_{1}, x_{2}\right\rangle^{\perp}$, then $\langle z\rangle$ is on two axis lines, by Lemma 4 . Thus

$$
n=\left\langle x_{-1}+c x_{1}+d x_{2}+v, x_{-2}+a x_{2}+b x_{1}+u\right\rangle
$$

where $v, u \in W^{\perp}, a, b, c, d \in F$.

Case 2. Suppose $a=0$. Then since $y=x_{-2}+b x_{1}+u$ is perpendicular to $\left\langle b x_{2}-x_{-1}, x_{-2}+b x_{1}\right\rangle=k$, an axis line for $H$, we must have $x=x_{-1}+c x_{1}+d x_{2}+v$ also in $k^{\perp}$, so that $y$ is not on two axis lines (Lemma 4). Hence $c=0$ and $d=-b$ and $n=\left\langle x_{-1}-b x_{2}+v, x_{-2}+\right.$ $b x_{1}+u>$.

Thus $u$ and $v$ are both singular, $u \in v^{\perp}$, and $\langle u, v\rangle \in W^{\perp}$. Thus the dimension of $V$ must be at least 8 . We return to this case later.

Case 1. Suppose $a \neq 0$. By Lemma 5 we may send $x_{-2}+a x_{2}+$ $b x_{1}+u$ to $z=x_{-2}+a x_{2}+u$ by an element of $H$.

Hence we get an axis line for $G, n=\langle w, z\rangle$. Choose $w$ on $n$ in $u^{\perp}$. Then $w=e x_{1}+f x_{-1}+g x_{2}+h x_{-2}+x$ where $x \in\langle W, u\rangle^{\perp}$. Hence $g+a h=0$ or $g=-a h$. At this point, we need more information. We thus note:

LEMMA 8 [6]. Using standard basis notation, if $l=\left\langle x_{1}, x_{2}\right\rangle$, $m=\left\langle x_{-1}, x_{-2}\right\rangle, n=\left\langle x_{-2}+a x_{2}+u, h x_{-2}-a h x_{2}+e x_{1}+f x_{-1}+x\right\rangle$ where $\langle x, u\rangle \in\langle l, m\rangle^{\perp}$ and $\langle x, u\rangle$ is anisotropic, then $\Sigma l, \Sigma m$, and $\Sigma$ n generate a group $G$ isomorphic to $S U_{3}(q)$. In addition, $G$ is transitive on the singular vectors in $V=\langle l, m, n\rangle$ and is transitive on the nonsingular vectors of a given length, and every singular point of $V$ is on an axis line for $G$.

By Lemma 8 , if $\langle u, x\rangle$ is anisotropic, the group generated by $H$ and $\Sigma n$ is isomorphic to $S U_{3}(q)$.

Now suppose $\langle u, x\rangle$ contains one singular point, $\langle y\rangle$. Then $\langle t+y\rangle \in n$ for some $t \in W$. But since $y$ is singular, $t$ is singular and hence $t$ belongs to some axis line $s$ of $H$. Thus $\langle t+y\rangle \in s^{\perp}$. But $n \cap s^{\perp} \neq n$ since $\langle n, W\rangle$ does not contain a 4-dimensional singular space. Thus by Lemma $4,\langle t+y\rangle$ must be on two axis lines for $G$, contradicting the hypothesis of this section. 
Lemma 9. Let $m$ and $l$ be axis lines for $G$ such that $m \cap l^{\perp}=\phi$. Let $\Sigma n$ move some point of $\langle m, l\rangle$ outside of $\langle m, l\rangle$. If we assume that for the group $T$ generated by $\Sigma m, \Sigma l$, and $\Sigma$ n, no two lines intersect, then either (a) $\langle m, l, n\rangle$ contains a 4-dimensional singular space (Case 2 above) or else (b) $T \cong S U_{3}(q)$ and $\langle m, l, n\rangle$ is nondegenerate with dimension 6 and index 2.

As a consequence of Lemma 9, we now prove

LEMma 10. Suppose no two axis lines of $G$ intersect. If $T \cong S U_{k}(q)$ is a subgroup of $G$ fixing a 2k-dimensional subspace $U$ of $V$, then either

(1) $U=V$,

(2) there is a Case 1 axis line moving $U$ and $G \supseteqq S U_{k+1}(q)$, or (3) there is no Case 1 axis line moving $U$; axis lines moving $U$ are Case 2 axis lines.

Proof. If $U \neq V, T$ is reducible on $V$ and there must be another axis line $h$. Thus $\Sigma h$ is a subgroup of $G$ which does not fix $U$ and for some pair of opposites $\Sigma l, \Sigma m$ in $T, \Sigma h$ does not fix $\langle l, m\rangle$. If $h$ is a "Case 1 " line, $\langle h, U\rangle \cap U^{\perp}$ has dimension 2 and is anisotropic. By work in [6], $\Sigma h$ corresponds to a unitary transvection with center $\langle x+y\rangle$ where $x$ is in $U$ and $y$ is nonisotropic in $U^{L}$. (We have abused notation by identifying the orthogonal geometry of $U$ and the corresponding unitary geometry of $U$.) One wishes to know that $S U_{k}(q)$ and such a unitary transvection generate $S U_{k+1}(q)$. But one need only remark that $S U_{k}(q)$ is transitive on nonisotropic vectors of a given length, to see that if $\langle x+y\rangle$ is a center of a transvection for $G$, then so is $(z+y)$ for any $z$ in $U$ of the same length. Thus $T$ and $\Sigma h$ generate a group isomorphic to $S U_{k+1}$, since such unitary groups are generated by the set of all unitary transvections.

6. No two axis lines of $G$ intersect (Case 2). We now assume that Case 1 does not occur in $G$. We have in $G$, the subgroup $H$, generated by the opposites $\Sigma l$ and $\Sigma m$. In addition, by Lemma 3, we have for each $c$ and $d$ not both zero the axis line

$$
n(c, d)=\left\langle c x_{-1}+d x_{2}+x_{3}, c x_{-2}-d x_{1}+x_{4}\right\rangle .
$$

But $H$ and $\Sigma n(c, d)$ fix $\left\langle x_{3}, x_{4}\right\rangle$. In order that $G$ be irreducible we need still another axis line $k$ such that $\Sigma k$ moves $\left\langle x_{3}, x_{4}\right\rangle$. (Hence $\Sigma k$ is opposite to $\Sigma n(c, d)$ for some $c, d$.)

First assume $k$ is in $W^{\perp}$. Then let $\left\langle x_{-4}\right\rangle=k \cap\left\langle x_{3}\right\rangle^{\perp}$ and let $\left\langle x_{-3}\right\rangle=k \cap\left\langle x_{4}\right\rangle^{\perp}$. Thus $k=\left\langle x_{-3}, x_{-4}\right\rangle$. 
If $k$ is not in $W^{\perp}$, there are two possibilities

(1) $k=l(c, d)=\left\langle c x_{1}+d x_{2}+x_{-3}, c x_{2}-d x_{-1}-x_{-4}\right\rangle$

(2) $k=p(c, d)=\left\langle c x_{1}+d x_{-2}+x_{-4}, c x_{2}-d x_{1}+x_{-3}\right\rangle$.

Since the group generated by $\Sigma l(c, d), \Sigma n(c, d)$, and $H$ is isomorphic to the group generated by $\Sigma\left\langle x_{-3}, x_{-4}\right\rangle, \Sigma n(c, d)$, and $H$, we may identify possibility (1) with $k=\left\langle x_{-3}, x_{-4}\right\rangle$.

Further, a line of type (2) in addition to a line of type $n(c, d)$ produces a Case 1 configuration in $G: \Sigma\left\langle x_{1}+x_{-4}, x_{2}+x_{-3}\right\rangle$ sends $\left\langle x_{-1}+x_{3}, x_{-2}+x_{4}\right\rangle$ to $\left\langle x_{-1}+x_{2}+x_{-3}+x_{3}-x_{1}-x_{-4}, \cdots\right\rangle$.

Thus, we may choose $k \in W^{\perp}$. We remark that the group $T$ generated by $\Sigma k, \Sigma n(c, d)$, and $H$ fixes the space $\left\langle x_{-1}, x_{2}, x_{3}, x_{-4}\right\rangle$.

We generalize this process:

Lemma 11. Suppose $G$ has no Case 1 configuration and $G$ contains a subgroup $T(n)(n \geqq 2)$ generated by groups of root type 1 with the following axis lines: $\left\langle x_{1}, x_{2}\right\rangle,\left\langle x_{-1}, x_{-2}\right\rangle, \cdots,\left\langle x_{-1}+x_{2 n-1}, x_{-2}+x_{2 n}\right\rangle$, $\left\langle x_{-(2 n-1)}, x_{-2 n}\right\rangle$. Then (1) $T(n)$ is transitive on the (singular) vectors in the space $X=\left\langle x_{-1}, x_{2}, x_{3}, x_{-4}, \cdots, x_{2 n-1}, x_{-2 n}\right\rangle$ and $T(n)$ fixes $X$.

(2) If $k$ is a singular line in $U$, the space spanned by axis lines of $T(n)$, and $\Sigma k$ is not an axis line for $T(n)$, then $\Sigma k$ is not an axis line for $G$.

(3) $G$ contains $T(n+1)$.

We remark that as a consequence of this lemma, since $V$ is finite dimensional, there can be no group $G$ without a Case 1 configuration.

The proof of (1) is tedious but straightforward. One shows all vectors in $\left\langle x_{-1}, x_{2}, x_{2 j-1}, x_{-2 j}\right\rangle 2 \leqq j \leqq n$ are in the same orbit by applying in sequence various transformations in $H, \Sigma\left\langle x_{-(2 j-1)}, x_{-2 j}\right\rangle$ and $\Sigma\left\langle x_{-1}+x_{2 j-1}, x_{-2}+x_{2 j}\right\rangle$. Then one remarks that $x_{-1}$ can be sent to an arbitrary vector of $X, a_{1} x_{-1}+a_{2} x_{2}+\cdots+a_{2 n} x_{-2 n}$ by sending $x_{-1}$ to $x_{-1}+a_{3} x_{3}+a_{4} x_{-4}$, then fixing $a_{3} x_{3}+a_{4} x_{-4}$ and sending $x_{-1}$ to $x_{-1}+$ $a_{5} x_{5}+a_{6} x_{-6}$, repeating until in the last place where $a_{2 j-1}$ and $a_{2 j}$ are not both zero, one sends $x_{-1}$ to $a_{1} x_{-1}+a_{2} x_{2}+a_{2 j-1} x_{2 j-1}+a_{2 j} x_{-2 j}$.

By part (1) every point in $X$ is on (exactly) one axis line for $T(n)$. A new axis line $k$ in $U$ must have no point in $X$. Since $U \cap X^{\perp}=X, k$ must not be on $X^{\perp}$. Without loss of generality, say $k=\left\langle x_{1}+y, z\right\rangle$ and let $z=k \cap\left\langle x_{-1}\right\rangle^{\perp}$. Then $z=x_{2}+w$, since $\Sigma k$ is an opposite to $\Sigma\left\langle x_{-1}, x_{-2}\right\rangle$. But $z \notin X$. Thus $w \notin X$. Thus there is a component of $z$ in $Y=\left\langle x_{1}, x_{-2}, x_{-3}, x_{4}, \cdots, x_{2 n}\right\rangle$. Say $b x_{2 j}$ is a component, then $\Sigma\left\langle x_{-1}+x_{-2 j}, x_{-2}+x_{-(2 j-1)}\right\rangle(\subseteq T(n))$ moves $z$ to $z+$ $\left(x_{-1}+x_{-2 j}\right)-b\left(x_{-2}+x_{-(2 j-1)}\right)$. But then we have a Case 1 configuration (if $b x_{-(2 j-1)}$ is a component, use $\Sigma\left\langle x_{-1}+x_{2 j-1}, x_{-2}+x_{2 j}\right\rangle$ ). This proves (2).

Thus we must have a line $r$ moving $X$ and lying outside of $U$. 
Without loss of generality, say $r$ moves $x_{-1}, r=\langle x+y, z+w\rangle$ where $x, z \in U$ and $y, w \notin U$. Say $z$ has an $x_{1}$ component. Say $\langle x+y\rangle \subseteq$ $\left\langle x_{-1}\right\rangle^{\perp} \cap r$. Then $x=x_{2}+a_{3} x_{3}+b_{3} x_{-3}+\cdots+a_{2 n} x_{2 n}+b_{2 n} x_{-2 n}$. Since $T(n)$ is transitive on vectors in $X$, and fixes $X$, some element in $T(n)$ sends $x$ to $y=x_{2}+c_{3} x_{-3}+c_{4} x_{4}+\cdots+c_{2 n-1} x_{-(2 n-1)}+c_{2 n} x_{2 n}$. Some $\sigma \in \Sigma\left\langle x_{-1}+x_{2 j-1}, x_{-2}+x_{2 j}\right\rangle$ sends $y$ to $y+c_{2 j-1}\left(-x_{-2}-x_{2 j}\right)+x_{-1}+x_{2 j-1}$. But since there are no Case 1 configurations, $c_{2 j-1}=0$ for all $j$. Further, some $\tau \in \Sigma\left\langle x_{-2 j}, x_{-(2 j-1)}\right\rangle$ sends $y$ to $y+c_{2 j}\left(x_{-(2 j-1)}\right)$. Thus $c_{2 j}=0$ as well, since the coefficient of $x_{-(2 j-1)}$ must be zero. Thus we may choose $x=x_{2}$, or without loss of generality $x$ can be chosen to be any vector in $X$, say $x=x_{-1}$. The hypothesis of no two axis lines intersecting yields $r=\left\langle x_{-1}+y, x_{-2}+w\right\rangle$ with $y, w \in U^{\perp}$. Without loss of generality $r=\left\langle x_{-1}+x_{2 n+1}, x_{-2}+x_{2 n+2}\right\rangle$. In addition, we have shown that an axis line of form $\langle x+y, z+w\rangle$ with $x, z \in U$ and $y, w \in U^{\perp}$ can be written with $x \in X$. Hence, if we are to have a line which does not fix $\left\langle x_{2 n+1}, x_{2 n+2}\right\rangle$, we must have lines of form $\left.\left\langle x_{-(2 n+1)}, x_{-(2 n+2}\right)\right\rangle$, or $\left\langle x_{-1}+x_{-(2 n+1)}, x_{-2}+x_{-2 n}\right\rangle$ as before. But the latter produces a Case 1 configuration. This proves part (3).

We now prove:

THEOREM 1. If no two axis lines of $G$ intersect, $G \cong S U_{k}(q)$, $k \geqq 3$. (In this case $\operatorname{dim} V=2 k$. If $k$ is even, index $V=k$. If $k$ is odd, index $V=k-1$.)

Proof. We have shown that if $G$ is a group satisfying the hypothesis of this paper, such that no two axis lines intersect, then $G$ must contain $S U_{3}(q)$. Let $n$ be the largest number such that $T \cong S U_{n}(q) \subseteq G$ and let $U$ be the $2 n$-dimensional space fixed by $T$. If $U=V$, we are done. If not, there must be an axis line $r$ for $G$ such that $\Sigma r$ does not fix $U$. Since $T$ is transitive on the singular vectors of $U$ (for this we need $n \geqq 3$ ) and since no two axis lines of $G$ intersect, we may write $r=\left\langle x_{-1}+x_{n+1}, x_{-2}+x_{n+2}\right\rangle$. (If $r$ were Case 1, we would contradict the maximality of $n$.) In addition we must have $\left\langle x_{-(n+1)}, x_{-(n+2)}\right\rangle$. But $T$ is transitive on the singular vectors of $U$. So we then also have as axis line $\left\langle x_{1}+x_{n+1}, x_{2}+x_{n+2}\right\rangle$, and $\Sigma\left\langle x_{-(n+1)}, x_{-(n+2)}\right\rangle$ sends $\left\langle x_{1}+x_{n+1}, x_{2}+x_{n+2}\right\rangle$ to

$$
k=\left\langle x_{1}+x_{n+1}+x_{-(n+2)}, x_{2}+x_{n+2}-x_{-(n+1)}\right\rangle
$$

and $\Sigma k$ sends $r$ to $\left\langle x_{-1}+x_{2}+x_{n+2}-x_{-(n+1)}+x_{n+1}+x_{1}+x_{n+1}+x_{-(n+2)}\right.$, $\cdots\rangle$, a Case 1 configuration.

7. Two axis lines contain the same point and are perpendicular to each other. If two axis lines $l$ and $m$ for $G$ contain the same point $P$, then either $l$ is perpendicular to $m$ (e.g., $\left\langle x_{1}, x_{2}\right\rangle$ and 
$\left.\left\langle x_{1}, x_{3}\right\rangle\right)$ or else $l \cap m^{\perp}=P$ (e.g., $\left\langle x_{1}, x_{2}\right\rangle$ and $\left.\left\langle x_{1}, x_{-2}\right\rangle\right)$. We suppose in this section that the former case occurs, but the latter does not, and in addition for no point $Q$ is the space of axis lines containing $Q$ of dimension larger than 3 .

Let $P=\left\langle x_{1}\right\rangle$. Using standard basis notation we assume $\left\langle x_{1}, x_{2}\right\rangle$ is an axis line containing $P$. Let the space spanned by the axis lines containing $P$ be called $S$.

Not that the hypotheses of this section imply $S$ is singular.

Let an opposite to $\left\langle x_{1}, x_{2}\right\rangle$ be denoted $\left\langle x_{-1}, x_{--2}\right\rangle$ as usual. Then $l=S \cap\left\langle x_{-2}\right\rangle^{\perp}$ has dimension 2 and includes $x_{1}$. By the following lemma, $l$ is an axis line for $G$.

LEMMA 12. If $S$ is the space spanned by axis lines for $G$ which contain the singular point $P$, and $P \subseteq m \subseteq S$, where $m$ is a line, then $m$ is an axis line for $G$.

Proof. Say $l_{i}=\left\langle P, x_{i}\right\rangle 1 \leqq i \leqq k$ are the axis lines spanning $S$. Then $m=\left\langle P, \sum_{i=1}^{k} c_{i} x_{i}\right\rangle$. By the fact that $\rho_{x, u} \rho_{x, \nu}=\rho_{x, u+\nu}$, we see that $m$ is an axis line for $G$. (If $S$ is not singular, $\Sigma m$ may be a group of root type 2.)

Thus using standard notation, we may name our $l$ above " $\left\langle x_{1}, x_{3}\right\rangle$ " where $x_{3} \in\left\langle x_{-2}, x_{-1}, x_{2}, x_{1}\right\rangle$.

We remark that $\Sigma\left\langle x_{-1}, x_{-2}\right\rangle$ sends $\left\langle x_{1}, x_{3}\right\rangle$ to $\left\langle x_{1}+a x_{-2}, x_{3}\right\rangle$. By Lemma 12, $\left\langle x_{-2}, x_{3}\right\rangle$ is also an axis line for $G$. By Baer's theorem (Lemmas 6 and 7), we know that $\left\langle x_{1}, x_{3}\right\rangle$ must have an opposite; call it $m$.

Let $m=\langle x+w, y\rangle$ where $w+x \in x_{3}^{\perp}$ and $y \in x_{1}^{\perp}$, and $w \in\left\langle x_{1}, x_{2}\right.$, $\left.x_{-1}, x_{-2}\right\rangle^{\perp}$. Thus $x=x_{-1}+a x_{1}+b x_{2}+c x_{-2}$ for some $a, b, c \in F$.

Case A. Assume $w$ is singular or zero. Hence $x$ is singular. Let $H$ be the group generated by $\Sigma\left\langle x_{1}, x_{2}\right\rangle$ and $\Sigma\left\langle x_{-1}, x_{-2}\right\rangle$. Since $x$ is singular we can find a $\sigma \in H$ which sends $m$ to $\sigma(m)=\left\langle x_{2}+d x_{1}+\right.$ $w, \sigma(y)\rangle$ where $d \in F$. (Note that since the coefficient of $x_{-1}$ is nonzero in $x$, the coefficient of $x_{2}$ is nonzero in $\sigma(x)$. Further, note that $\sigma(y)$ must contain an " $x_{-3}$ " component since $y$ did, and $\sigma \in H$.)

If $\sigma(y)$ is perpendicular to $\left\langle x_{1}, x_{2}\right\rangle$, then $\Sigma \sigma(m)$ sends $\left\langle x_{1}, x_{3}\right\rangle$ to $\left\langle x_{1}, x_{3}+x_{2}+d x_{1}+w\right\rangle$. Thus $w \in\left\langle x_{3}\right\rangle$. However we show $w \in\left\langle x_{3}\right\rangle$ even if $\sigma(y)$ is not perpendicular to $\left\langle x_{1}, x_{2}\right\rangle$.

If $\sigma(y)$ is not perpendicular to $\left\langle x_{1}, x_{2}\right\rangle$, then by Lemma $4,\left\langle x_{2}+\right.$ $\left.d x_{1}+w\right\rangle$ is on an axis line with some point $P$ on $\left\langle x_{1}, x_{2}\right\rangle$. Since $\sigma(y)$ is not perpendicular to $x_{3},\left\langle x_{2}+d x_{1}+w\right\rangle$ is on an axis line with some point $Q$ in $\left\langle x_{1}, x_{3}\right\rangle$. Thus $\left\langle x_{2}+d x_{1}+w\right\rangle$ is on axis lines with $P, Q$, and $\sigma(y)$. If $P \neq Q$, these three points are linearly independent and $\left\langle x_{2}+d x_{1}+w\right\rangle$ is on too many linearly independent axis lines for the hypothesis of this section. Thus $P=Q=\left\langle x_{1}\right\rangle$. But then $\left\langle x_{1}\right\rangle$ is on 
too many axis lines, unless $w \in\left\langle x_{3}\right\rangle$.

Therefore, we may suppose that $\sigma(m)=\left\langle x_{2}+d x_{1}+c x_{3}, x_{-3}+a x_{-2}+\right.$ $\left.b x_{1}+e x_{2}+f x_{3}+z\right\rangle$ where $z \in\left\langle x_{1}, x_{2}, x_{3}, x_{-1}, x_{-2}, x_{-3}\right\rangle^{\perp}$, and there is no $x_{-1}$ component since $\left\langle x_{2}+d x_{1}+c x_{3}, x_{1}\right\rangle$ is an axis line. In addition, by Lemma 12 , we may assume $b=0$.

There is an element $t \in \Sigma\left\langle x_{-2}, x_{3}\right\rangle$ which sends $\sigma(m)$ to $t \sigma(m)=$ $\left\langle x_{2}+d x_{1}, x_{-3}+h x_{-2}+e x_{2}+g x_{3}+z\right\rangle$. Since $t \sigma(m)$ is singular, $h=0$. By Lemma 12 we may also assume $e=0$, and we obtain $t \sigma(m)=$ $\left\langle x_{2}+d x_{1}, x_{-3}+g x_{3}+z\right\rangle$. Here $d$ may be zero or not.

Let $T$ be the group generated by $H, \Sigma\left\langle x_{1}, x_{3}\right\rangle$, and $\Sigma m$ for Case A. But $T$ fixes the space $U=\left\langle x_{1}, x_{-2}, x_{-3}+g x_{3}+z\right\rangle$, so $T$ is not irreducible. Further, $T$ is transitive on the vectors of $U$. Thus we have an axis line for $G$ which moves $x_{1}$, say $l=\left\langle x_{-1}+y, w\right\rangle$ where $w \notin U$. Let $w$ be in $x_{1}^{\perp}$. If $w$ is also in $x_{2}^{\perp}, w$ is on an axis line with some point in $\left\langle x_{1}, x_{2}\right\rangle$, not $\left\langle x_{1}\right\rangle$. If $w$ is also in $x_{3}^{\perp}, w$ is on an axis line with still another point. Further, $\left\langle w, a x_{1}+x_{2}, b x_{1}+x_{3}\right\rangle$ must be 3-dimensional since no point in $\left\langle x_{1}, x_{2}, x_{3}\right\rangle$ can be on an axis line with $x_{-1}+y$, these all being on axis lines with $\left\langle x_{1}\right\rangle$. Hence $\left\langle w, a x_{1}+\right.$ $\left.x_{2}, b x_{1}+x_{3}, x_{-1}+y\right\rangle$ (in the space of axis lines containg $w$ ) is 4-dimensional. This implies $w$ must either not be perpendicular to $x_{2}$ or else not to $x_{3}$.

Suppose $w$ is not perpendicular to $x_{2}$, but is perpendicular to $x_{3}$. Then $w=a x_{1}+b x_{2}+x_{-2}+c x_{3}+x$ where $x \in\left\langle x_{1}, x_{2}, x_{3}, x_{-1}, x_{-2}, x_{-3}+\right.$ $\left.g x_{3}+z\right\rangle^{\perp}$ and $w$ is on an axis line with $x_{1}+d x_{3}, d \neq 0$. We may use Lemma 12 with $\left\langle x_{1}+d x_{3}, x_{3}\right\rangle$ and $\left\langle x_{1}+d x_{3}, x_{1}\right\rangle$ to get the axis line $m=\left\langle x_{1}+d x_{3}, b x_{2}+x_{-2}+x\right\rangle$. If $b=0, \Sigma m$ sends $\left\langle x_{-2}, x_{-1}\right\rangle$ to $\left\langle x_{-2}, x_{-1}+x_{-2}+x\right\rangle$. But then $x_{-2}$ is on too many axis lines (since $x$ is linearly independent of $x_{-1}$ and $x_{3}$ ). Thus $b \neq 0$. But by [6], this gives us a $G_{2}$ configuration in the 7 -dimensional space $\left\langle x_{1}, x_{2}, x_{-1}, x_{-2}\right.$, $\left.x_{3}, x_{-3}+g x_{3}+z, x\right\rangle$.

If $w$ is not perpendicular to $x_{3}$, we have another opposite to $\left\langle x_{1}, x_{3}\right\rangle$. If we are in Case B (which follows) we end up with a line $m\left(=\left\langle x_{1}+d x_{3}, x_{-2}+b x_{2}+x\right\rangle\right)$ and $G_{2}$, as above. So we assume Case $A$ again occurs and we have a line of form $l=\left\langle x_{-1}+a x_{-2}, x_{-3}+\right.$ $\left.f x_{3}+x\right\rangle$, where $x_{-3}+f x_{3}+x \notin\left\langle x_{-3}+g x_{3}+z\right\rangle$ since $w \notin U$, and $U$ is fixed by $T$. But $x_{-3}+f x_{3}+x \in\left\langle x_{1}, x_{2}, x_{-1}, x_{-2}\right\rangle^{\perp \cdot}$ If $x_{-3}+f x_{3}+x \epsilon$ $\left\langle x_{-3}+g x_{3}+z\right\rangle^{\perp}$ then $x_{-3}+f x_{3}+x$ is on axis lines with $x_{-1}-d x_{-2}$ and $x_{2}+d x_{1}$, as is $x_{-3}+g x_{3}+z$. Whether or not $d=a, x_{2}+d x_{1}$ ends up on too many axis lines. Thus $\left\langle x_{-3}+f x_{3}+x\right\rangle \notin\left\langle x_{-3}+g x_{3}+z\right\rangle^{\perp}$ and again by [6], and Witt's theorem, we get $G_{2}$.

Case B. We recall that $\left\langle x_{1}, x_{2}\right\rangle,\left\langle x_{-1}, x_{-2}\right\rangle,\left\langle x_{1}, x_{3}\right\rangle$, and $\left\langle x_{-2}, x_{3}\right\rangle$ are axis lines for $G$. Further $\Sigma m$ is an opposite to $\Sigma\left\langle x_{1}, x_{3}\right\rangle$, and $m=\langle x+w, y\rangle$, where $\langle y\rangle=m \cap\left\langle x_{1}\right\rangle^{\perp},\langle x+w\rangle=m \cap\left\langle x_{3}\right\rangle^{\perp}$, and 
$w \in\left\langle x_{1}, x_{2}, x_{-1}, x_{-2}\right\rangle^{\perp}$. In Case $\mathrm{B}, w$ is nonsingular.

By Lemma $5, H$, the group generated by $\Sigma\left\langle x_{1}, x_{2}\right\rangle$ and $\Sigma\left\langle x_{-1}, x_{-2}\right\rangle$ is transitive on nonsingular vectors of a given length. Thus we may assume for $a \neq 0, m=\left\langle x_{-2}+a x_{2}+w, y\right\rangle$ where $y$ has an $x_{-3}$ component.

If $w=b x_{3}+z$, where $z \in\left\langle x_{3}, x_{-3}\right\rangle^{\perp}$, then apply $\sigma \in \Sigma\left\langle x_{-2}, x_{3}\right\rangle$ to obtain $\sigma(m)=\left\langle x_{-2}+a x_{2}+z, x\right\rangle$. We may choose $x=\sigma(m) \cap z^{\perp}$. Again, $x \notin x_{3}^{\perp}$, so we may use Lemma 4 to see $x_{-2}+a x_{2}+z$ is on an axis line with some point $P$ in $\left\langle x_{1}, x_{3}\right\rangle$. We obtain $l=\left\langle x_{-2}+a x_{2}+\right.$ $\left.z, x_{1}+b x_{3}\right\rangle$ where $b$ is not zero, for otherwise $x_{1}$ is on too many axis lines. (Since $x_{3}$ is on an axis line with $x_{-2}, P \neq\left\langle x_{3}\right\rangle$.)

We recall that $\sigma(m)\left(=\left\langle x_{-2}+a x_{2}+z, x\right\rangle\right)$ is an axis line for $G$, and $x \notin x_{3}^{\perp}, x \in\left\langle x_{1}+b x_{3}\right\rangle^{\perp} \cap z^{\perp}$. Thus $x=x_{-3}-b x_{-1}+c x_{2}+d x_{3}+e x_{1}+$ $f x_{-2}+t$ where $t \in\left\langle x_{1}, x_{2}, x_{3}, x_{-1}, x_{-2}, x_{-3}, z\right\rangle^{\perp}$. By Lemma 12, there is an axis line $n=\left\langle x_{-2}+a x_{2}+z, x_{-3}-b x_{-1}+c x_{2}+g x_{3}+f x_{-2}+t\right\rangle$. Since $n$ is singular, $f=-a^{-1} c$. Look at the point $P=n \cap x_{-2}^{\perp}=n \cap$ $\left\langle x_{-1}, x_{-2}\right\rangle^{\perp} . \quad P=\left\langle x_{-3}-b x_{-1}+f x_{-2}+g x_{3}+t-a^{-1} c x_{-2}-a^{-1} c z\right\rangle=$ $\left\langle x_{-3}-b x_{-1}-2 a^{-1} c x_{-2}+g x_{3}+t-a^{-1} c z\right\rangle$. But $P$ is on an axis line with some point in $\left\langle x_{-1}, x_{-2}\right\rangle$, as well as with $x_{-2}+a x_{2}+z$. Thus $P$ is on an axis line with $\left\langle x_{-1}\right\rangle$. Apply Lemma 12 to see $r=\left\langle x_{-1}, x_{-3}+\right.$ $\left.g x_{3}+t-a^{-1} c z\right\rangle$ is an axis line. By [6], the group generated by $\Sigma r, \Sigma l, H$, and $\Sigma\left\langle x_{1}, x_{3}\right\rangle$ is isomorphic to $G_{2}$ on the 7-dimensional space $\left\langle x_{1}, x_{2}, x_{-1}, x_{-2}, x_{3}, x_{-3}+g x_{3}+t-a^{-1} c z, z\right\rangle$. We remark for the convenience of the reader that in [6], we showed that $G_{2}$ is generated by $\Sigma\left\langle x_{1}, x_{2}\right\rangle, \Sigma\left\langle x_{-1}, x_{-2}\right\rangle, \Sigma\left\langle x_{1}, x_{3}\right\rangle, \Sigma\left\langle x_{-1}, x_{-3}\right\rangle, \Sigma\left\langle x_{2}, x_{-3}\right\rangle, \Sigma\left\langle x_{-2}, x_{3}\right\rangle$ and $\Sigma\left\langle x_{1}+d x_{3}, b x_{2}+x_{-2}+x\right\rangle$ where $x \in\left\langle x_{1}, x_{2}, x_{-1}, x_{-2}, x_{3}, x_{-3}\right\rangle^{\perp}$. In Case A, " $x_{-3}$ " was replaced with " $x_{-3}+g x_{3}+z$ ". (Witt's theorem makes this substitution legal.) In Case $B$, " $x_{-3}$ " was replaced by " $x_{-3}+$ $g x_{3}+t-a^{-1} c z$ " and " $x$ " by " $z+k x_{3}$ " where $k$ is chosen such that $z+k x_{3} \in\left\langle x_{-3}+g x_{3}+t-a^{-1} c z\right\rangle^{\perp}$. Then note we have the line $\left\langle x_{-2}+\right.$ $\left.a x_{2}+z+k x_{3}, x_{1}+b x_{3}\right\rangle$ as an axis line for $G$ by Lemma 12.

Thus we have seen we have (under the hypotheses of this section) a $G_{2}$ formation, which by [6] is transitive on the singular points of the 7-dimensional space $U$ spanned by the axis lines. Suppose then that $G$ contains $G_{2}$ and acts irreducibly on a space of dimension larger than 7 .

Thus, without loss of generality we have an axis line $l=\left\langle x_{-1}+\right.$ $u, \nu\rangle$ where no point on $l$ is in $U$, since all the singular points in $U$ are already on the required number of axis lines. Let $\nu$ be the point on $l$ in $x_{1}^{\perp}$. But $l$ must be opposite to all lines of form $\left\langle x_{1}, a x_{2}+b x_{3}\right\rangle$, $a$ and $b$ not both zero, or by Lemma 4 some point on one of these lines will be on an axis line with $\nu$. This is impossible since $\nu$ must 
be perpendicular to some point on $\left\langle x_{2}, x_{3}\right\rangle$. This establishes:

THEOREM 2. The irreducible subgroups $G$ of $\Omega(V)$ generated by groups of root type 1 such that (1) two axis lines $l$ and $m$ contain the same point $P$ and $l \subseteq m^{\perp}$, but no two axis lines intersect which are not perpendicular to each other and (2) for no point $Q$ is the space of axis lines of dimension larger than 3 , are the groups $G_{2}(q)$. The dimension of $V$ is 7 in this case.

8. The nondegenerate axis line space. Let $S$ be a maximal dimension space spanned by axis lines containing any one singular point. If we name this point $\left\langle x_{1}\right\rangle$, then let $l=\left\langle x_{1}, x_{2}\right\rangle$ be one of the axis lines in $S\left(=S\left(x_{1}\right)\right)$.

Suppose one of the opposites to $l$ is $m=\left\langle x_{-1}, x_{-2}\right\rangle$. Then $T=$ $S \cap\left\langle x_{-1}\right\rangle^{\perp}$. Suppose in this section that $T$ is nondegenerate.

Let $W=T \cap\left\langle x_{-2}\right\rangle^{\perp}$. We are going to work with $W$ and $\left\langle x_{-2}, x_{-1}\right\rangle$.

Let $w \in W$. Then $\rho_{x_{1}, w}$ sends $\left\langle x_{-2}, x_{-1}\right\rangle$ to $\left\langle x_{-2}, x_{-1}-Q(w) x_{1}-w\right\rangle$. Let $z=Q(w) x_{1}+w$. Then $\rho_{x_{-2}, z}$ sends $\left\langle x_{1}, x_{2}\right\rangle$ to $\left\langle x_{1}, x_{2}-Q(z) x_{-2}-z\right\rangle$. If $z$ (and hence $w$ ) is nonsingular, then one obtains $\left\langle x_{1}, x_{-2}\right\rangle$ as an axis line. In both cases ( $w$ singular or nonsingular), applying Lemma 12 to $\left\langle x_{-2}, x_{-1}-Q(w) x_{1}-w\right\rangle$ and $\left\langle x_{-2}, x_{-1}\right\rangle$ and (if $w$ is nonsingular) $\left\langle x_{-2}, x_{1}\right\rangle$, one obtains $\left\langle x_{-2}, w\right\rangle$ is an axis line for $G$.

If there are no nonsingular vectors in $W$, we may still obtain $\left\langle x_{-2}, x_{1}\right\rangle$ as an axis line as follows: We know every point in $W$ is on an axis line with $\left\langle x_{-2}\right\rangle$. In addition, $\left\langle x_{-1}\right\rangle$ is on an axis line with $\left\langle x_{-2}\right\rangle$, and $W \subseteq\left\langle x_{1}, x_{-1}\right\rangle^{\perp}$. If $x_{-2} \notin W$, then the geometry of the space of axis lines containing $\left\langle x_{-2}\right\rangle$ is different from the geometry of $S\left(x_{1}\right)$, (i.e., $S\left(x_{-2}\right)$ has dimension equal to $\operatorname{dim} S\left(x_{1}\right)$ but $T\left(x_{-2}\right)$ is degenerate, since $\left.x_{-1} \subseteq S\left(x_{-2}\right) \subseteq x_{-1}^{\perp}\right)$.

But the group generated by $\Sigma\left\langle x_{1}, x_{2}\right\rangle$ and $\Sigma\left\langle x_{-1}, x_{-2}\right\rangle$ sends $x_{1}$ to $x_{-2}$, so this is impossible. Thus $\left\langle x_{1}, x_{-2}\right\rangle$ is an axis line.

We have thus obtained:

LEMmA 13. If $S(x)$ is the space of axis lines containing $x$, and $m$ is opposite to $l$, one of those axis lines ( $m$ and $l$ both singular), then $S\left(m \cap x^{\perp}\right) \supseteqq\left(S(x) \cap m^{\perp}\right) \oplus m$. If in addition $T(x)$ is nondegenerate, or if $S(x) \cap m^{\perp}$ contains a nonsingular point, then $m \cap x^{\perp}$ is on an axis line with $x$, and $\langle m, S(x)\rangle=\left\langle l, S\left(m \cap x^{\perp}\right)\right\rangle$.

Let $n=\left\langle x_{-1}+u, x_{2}+v\right\rangle$ be an opposite for $\left\langle x_{1}, x_{-2}\right\rangle$, where $v, u \in\left\langle x_{1}, x_{-2}\right\rangle^{\perp}$. Let $T\left(x_{1}\right)=\left\langle x_{2}, x_{-2}, U\right\rangle$ where $U \subseteq\left\langle x_{1}, x_{-1}, x_{-2}, x_{2}\right\rangle^{\perp}$.

Using Lemma 13, we obtain that $\left\langle x_{2}+v\right\rangle$ must be on an axis line with $x_{1}$, so that $v=a x_{1}+b x_{-2}+w, w \in U\left(x_{1}\right)$. A similar argument using $S\left(x_{-2}\right)$ (instead of $S\left(x_{1}\right)$ ) shows that $x_{-1}+u$ must be on an 
axis line with $x_{-2}$, so that in particular $u \in\left\langle S\left(x_{-2}\right)\right\rangle,\left(u=d x_{1}+c x_{-2}+\right.$ $\left.x, x \in U\left(x_{1}\right)\right)$. Let $Z=\left\langle S\left(x_{1}\right), x_{-1}\right\rangle$. We have shown $Z=\left\langle S\left(x_{-1}+u\right)\right.$, $\left.x_{1}\right\rangle=\left\langle S\left(x_{2}+v\right), x_{-2}\right\rangle=\left\langle S\left(x_{-2}\right), x_{2}\right\rangle$. We show, as in [6], that $\Omega(Z) \leqq G$.

Let $l=\langle x, y\rangle$ be a singular line in $Z$. If $\left\langle x_{2}+v\right\rangle$ or $\left\langle x_{-2}\right\rangle$ are on $l$, then $l$ is an axis line for $G$ by Lemma 12 . If not:

Case 1. $\langle x, y\rangle \in\left\langle x_{2}+v, x_{-2}\right\rangle^{\perp}$. Here $\left\langle x_{2}+v, x\right\rangle$ and $\left\langle x_{-2}, y\right\rangle$ are axis lines, so by Lemma 4 , so is $\langle x, y\rangle$.

Case 2. $\langle x\rangle \in\left\langle x_{2}+v, x_{-2}\right\rangle^{\perp},\langle y\rangle \in\left\langle x_{-2}\right\rangle^{\perp}$, (by symmetry, $y \in\left\langle x_{2}+\right.$ $v\rangle^{\perp}$ could be used instead). Then $\left\langle x, x_{-2}\right\rangle$ is an axis line and $\left\langle x_{-2}, y\right\rangle$ is an axis line where $k x_{-2}+y \in\left\langle x_{2}+v\right\rangle^{\perp}$. By Lemma $4,\left(x, k x_{-2}+y\right)$ is an axis line and by Lemma $12,\langle x, y\rangle$ is an axis line.

Case 3. $\langle x, y\rangle \in\left\langle x_{-2}\right\rangle^{\perp}$ (resp. $\left.\langle x, y\rangle \in\left\langle x_{2}+v\right\rangle^{\perp}\right)$. Since some point on the line $l$ will be perpendicular to $\left\langle x_{2}+v\right\rangle\left(\right.$ resp. $\left.\left\langle x_{-2}\right\rangle^{\perp}\right)$ this reduces to Case 2.

Case 4. $\langle x\rangle \in\left\langle x_{-2}, x_{2}+v\right\rangle^{\perp}$ but $\langle y\rangle \notin\left\langle x_{-2}\right\rangle^{\perp}, y \notin\left\langle x_{2}+v\right\rangle^{\perp}$ but $y=a\left(x_{2}+v\right)+b\left(x_{-2}\right)+w$ where $w \in\left\langle x_{-2}, x_{2}+v\right\rangle^{\perp}$. But by Case 1, $\langle x, w\rangle$ is an axis line. Thus since $\langle x, w\rangle,\left\langle x, x_{-2}\right\rangle$, and $\left\langle x, x_{2}+v\right\rangle$ are axis lines, Lemma 12 implies $\langle x, y\rangle$ is an axis line.

There are no more cases, because $Z$ is nondegenerate, so if $l$ is a singular line in $Z$ and $m$ is a hyperbolic line in $Z$ it cannot happen that $Z \cap m^{\perp} \cap l$ is $\{0\}$. (If this were true $Z=m^{\perp} \oplus l, m^{\perp}$ nondegenerate and $Z$ nondegenerate.) Thus $\Omega(Z) \subseteq G$. In addition, we have proved:

LEMmA 14. Let $Z$ be nondegenerate. Then the group generated by all groups of root type $1\left\{\Sigma\langle x, y\rangle \mid y \in x^{\perp} \cap Z\right\}$ and $\{\Sigma\langle z, w\rangle \mid w \in$ $\left.z^{\perp} \cap Z\right\}$ where $\langle x, z\rangle$ is some fixed hyperbolic line, is $\Omega(Z)$.

Now we wish to show $\Omega(Z)=G$. Clearly, so far $Z$ is fixed so that if $\Omega(Z) \neq G, G$ is reducible. Since the axis lines of $\Omega(Z)$ containing one point $P$ in $Z$ span a space which by hypothesis has maximal dimension, there can be no axis lines with one point in $Z$ and one point outside $Z$.

Without loss of generality, assume $l=\left\langle x_{-1}+u, v\right\rangle$ is an axis line, with no point on $l$ in $Z$. Let $v$ be the point on $l$ in $x_{1}^{\perp}$. If $l$ is opposite some line in $S\left(x_{1}\right)$, use Lemma 13. If not, $v \in S\left(x_{1}\right)^{\perp}$. Then $v$ is on axis lines with every point in $S\left(x_{1}\right) \cap\left\langle x_{-1}+u\right\rangle^{\perp}$ yielding a contradiction. Thus we have:

THEOREM 3. The irreducible groups generated by groups of root type 1 with nondegenerate axis line spaces are the groups $\Omega(V)$. 
9. The elimination of other possibilities: I. Mixed axis line spaces. We again suppose $S\left(x_{1}\right)$ is the space spanned by axis lines containing $x_{1}$ and that $S\left(x_{1}\right)$ is of maximal dimension. Let $S\left(x_{1}\right)=$ $\left\langle x_{1}\right\rangle \oplus T\left(x_{1}\right)$ indicating only that $x_{1}$ is linearly independent of $T\left(x_{1}\right)$. In the last section, we showed that if $T\left(x_{1}\right)$ were nondegenerate, $G=\Omega(V)$. In this section we assume $T$ is neither singular nor nondegenerate. Thus $T=U \oplus W$, where $U$ is a maximal nondegenerate subspace of $T$ and $W$ is singular and $W \subseteq U^{\perp}$, and $U$ and $W$ are both nonzero.

Some set of singular vectors spans $T$. They cannot all be pairwise perpendicular, or $U$ would be empty. Thus there are singular vectors $x, y$ such that $\langle x, y\rangle$ is hyperbolic and $\langle x, y\rangle \subseteq U$. Say $\langle x\rangle=x_{2}$. Then suppose $\left\langle x_{-1}, x_{-2}\right\rangle$ is an opposite to $\left\langle x_{1}, x_{2}\right\rangle$. Choose $T\left(x_{1}\right)$ in $\left\langle x_{-1}\right\rangle^{\perp}$ as before. Then $y=x_{-2}+a x_{2}+z$, where $z \in\left\langle x_{1}, x_{2}, x_{-1}, x_{-2}\right\rangle^{\perp}$.

As in the previous section, since the group generated by $\Sigma\left\langle x_{-1}, x_{-2}\right\rangle$ sends $x_{1}$ to $x_{-2}$, the geometries of their axis line spaces must be the same. But if we apply Lemma 13 to the lines containing $x_{1}$ which are in $T\left(x_{1}\right) \cap x_{\lrcorner_{2}}^{\perp}$ we get $\left\langle T\left(x_{1}\right) \cap x_{-2}^{\perp}, x_{-1}\right\rangle \subseteq S\left(x_{-2}\right)$. If $x_{-2} \notin T\left(x_{1}\right) \cap x_{-2}^{\perp}$, the geometry of $T\left(x_{-2}\right)$ is different from the geometry of $T\left(x_{1}\right)$. Thus we may assume that $\left\langle x_{1}, x_{-2}\right\rangle$ is an axis line.

As in the previous section, if $n=\left\langle x_{-1}+u, x_{2}+v\right\rangle$ is an opposite for $\left\langle x_{1}, x_{-2}\right\rangle$, then $\left\langle S\left(x_{1}\right), x_{-1}\right\rangle=\left\langle S\left(x_{-2}\right), x_{2}\right\rangle=\left\langle S\left(x_{-1}+u\right), x_{1}\right\rangle=\left\langle S\left(x_{2}+\right.\right.$ $\left.v), x_{-2}\right\rangle$. Note that if $x_{3} \in W\left(x_{1}\right)$, the singular part of the axis line space, then $\left\langle S\left(x_{1}\right), x_{-1}\right\rangle \subseteq x_{3}^{\perp}$. Then we see that $S\left(x_{-1}+u\right) \cap x_{3}^{\perp}=$ $S\left(x_{-1}+u\right)$. Now apply Lemma 4 to $\left\langle x_{1}, x_{3}\right\rangle$ and all the axis lines containing $x_{-1}+u$ to see $x_{3}$ is on axis lines with all of the points in $S\left(x_{-1}+u\right)$ and with $x_{1}$. Thus the space $S\left(x_{3}\right)$ has larger dimension, producing a contradiction.

10. The elimination of other possibilities: II. Singular axis line spaces of large dimension. We again let $\left\langle x_{1}, x_{2}\right\rangle$ be an axis line. We assume $\left\langle x_{-1}, x_{-2}\right\rangle$ is an opposite, and we let $S\left(x_{1}\right)$ be maximal dimension. In this section $T\left(x_{1}\right)=S\left(x_{1}\right) \cap x_{-1}^{\perp}$ and $T\left(x_{1}\right)$ is singular of dimension at least 3 . We name the vectors spanning $T\left(x_{1}\right)$ " $x_{2}, x_{3}$, $\cdots, x_{k}$ " according to standard basis notation. We obtain $T\left(x_{-2}\right)=$ $\left\langle x_{-1}, x_{3}, \cdots, x_{k}\right\rangle$ by repeated applications of Lemma 4 with $\left\langle x_{-1}, x_{-2}\right\rangle$. Let $\left\langle x_{-3}+u, x_{-1}+v\right\rangle$ be an opposite to $\left\langle x_{1}, x_{3}\right\rangle$ with $x_{-3}+u$ chosen in $x_{1}^{\perp}, x_{-1}+v$ chosen in $x_{3}^{\perp}$. Then $x_{-1}+v$ is on axis lines with all the points in $S\left(x_{1}\right) \cap\left(x_{-1}+v\right)^{\perp} \cap\left(x_{-3}+u\right)^{\perp}$ as well as with $x_{-3}+u$.

Now apply Lemma 4 with $\left\langle x_{1}, x_{3}\right\rangle$ to see $x_{3}$ is on axis lines with $S\left(x_{-1}+v\right) \cap x_{1}^{\perp} \cap x_{3}^{\perp}=S\left(x_{1}\right) \cap\left(x_{-1}+v\right)^{\perp} \cap\left(x_{-3}+u\right)^{\perp} \quad$ since $\quad S\left(x_{1}\right) \subseteq$ $\left\langle x_{1}, x_{3}\right\rangle^{\perp}$, as well as with $x_{1}$, and $x_{-2}$. But $R=\left\langle x_{1}, x_{2}, x_{3}\right\rangle \cap\left\langle x_{-1}+v\right\rangle^{\perp} \cap$ $\left\langle x_{-3}+u\right\rangle^{\perp}$ is nonempty. But $a x_{1}+b x_{3} \notin\left\langle x_{-1}+v\right\rangle^{\perp} \cap\left\langle x_{-3}+u\right\rangle^{\perp}$ for any $a, b$ not both zero. Thus $x_{2}+c x_{1}+d x_{3} \in R$. Thus $S\left(x_{3}\right)$ is not 
singular, but has maximal dimension. This reduces us to the case of the previous section, providing a contradiction. This completes the main theorem.

\section{REFERENCES}

1. E. Artin, Geometric Algebra, Interscience Publishers, Inc., 1965.

2. M. Aschbacher, A characterization of the unitary and symplectic groups over finite fields of characteristic at least 5, to appear.

3. B. Fischer, Finite groups generated by 3-transpositions I, Inventiones Math., 13 (1971), 232-246.

4. G. Glauberman, Quadratic elements in unipotent linear groups, J. Algebra, 20 (1972), 637-654.

5. D. Gorenstein, Finite Groups, Harper and Row, 1968.

6. B. Stark, Some subgroups of $\Omega(V)$ generated by groups of root type 1 , Illinois J. Math., 17 (1973), 584-607.

7. T. Tamagawa, On the structure of orthogonal groups, Amer. J. Math., 80 (1958), 191-197.

8. J. Thompson, Quadratic pairs, to appear.

Received April 24, 1973.

NORTHEASTERN UNIVERSITY 



\section{PACIFIC JOURNAL OF MATHEMATICS}

\section{EDITORS}

RICHARD ARENS (Managing Editor)

University of California

Los Angeles, California 90024

\section{J. DUGUNDJI}

Department of Mathematics University of Southern California Los Angeles, California 90007

D. Gilbarg and J. Milgram

Stanford University

Stanford, California 94305

University of Washington
Seattle, Washington 98105

ASSOCIATE EDITORS
E. F, BECKENBACH
B. H. NEUMANN
F. WOLF
K. Yoshida

\section{SUPPORTING INSTITUTIONS}

\author{
UNIVERSITY OF BRITISH COLUMBIA \\ CALIFORNIA INSTITUTE OF TECHNOLOGY \\ UNIVERSITY OF CALIFORNIA \\ MONTANA STATE UNIVERSITY \\ UNIVERSITY OF NEVADA \\ NEW MEXICO STATE UNIVERSITY \\ OREGON STATE UNIVERSITY \\ UNIVERSITY OF OREGON \\ OSAKA UNIVERSITY
}

\author{
UNIVERSITY OF SOUTHERN CALIFORNIA \\ STANFORD UNIVERSITY \\ UNIVERSITY OF TOKYO \\ UNIVERSITY OF UTAH \\ WASHINGTON STATE UNIVERSITY \\ UNIVERSITY OF WASHINGTON \\ * * * * \\ AMERICAN MATHEMATICAL SOCIETY \\ NAVAL WEAPONS CENTER
}

The Supporting Institutions listed above contribute to the cost of publication of this Journal, but they are not owners or publishers and have no responsibility for its content or policies.

Mathematical papers intended for publication in the Pacific Journal of Mathematics should be in typed form or offset-reproduced, (not dittoed), double spaced with large margins. Underline Greek letters in red, German in green, and script in blue. The first paragraph or two must be capable of being used separately as a synopsis of the entire paper. Items of the bibliography should not be cited there unless absolutely necessary, in which case they must be identified by author and Journal, rather than by item number. Manuscripts, in duplicate if possible, may be sent to any one of the four editors. Please classify according to the scheme of Math. Rev. Index to Vol. 39. All other communications to the editors should be addressed to the managing editor, or Elaine Barth, University of California, Los Angeles, California, 90024.

100 reprints are provided free for each article, only if page charges have been substantially paid. Additional copies may be obtained at cost in multiples of 50 .

The Pacific of Journal Mathematics is issued monthly as of January 1966. Regular subscription rate: $\$ 72.00$ a year (6 Vols., 12 issues). Special rate: $\$ 36.00$ a year to individual members of supporting institutions.

Subscriptions, orders for back numbers, and changes of address should be sent to Pacific Journal of Mathematics, 103 Highland Boulevard, Berkeley, California, 94708.

PUBLISHED BY PACIFIC JOURNAL OF MATHEMATICS, A NON-PROFIT CORPORATION

Printed at Kokusai Bunken Insatsusha (International Academic Printing Co., Ltd.), 270, 3-chome Totsuka-cho, Shinjuku-ku, Tokyo 160, Japan.

Copyright (C) 1973 by Pacific Journal of Mathematics Manufactured and first issued in Japan 


\section{Pacific Journal of Mathematics}

\section{Vol. 53, No. $2 \quad$ April, 1974}

Kenneth Abernethy, On characterizing certain classses of first countable spaces by

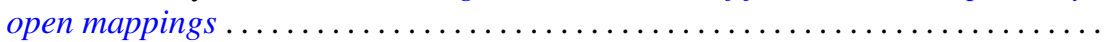

Ross A. Beaumont and Donald Lawver, Strongly semisimple abelian groups .......

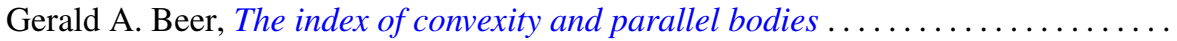

Victor P. Camillo and Kent Ralph Fuller, On Loewy length of rings ..............

Stephen LaVern Campbell, Linear operators for which $T^{*} T$ and $T T^{*}$ commute.

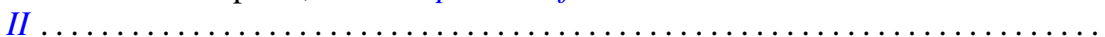

Charles Kam-Tai Chui and Philip Wesley Smith, Characterization of a function by

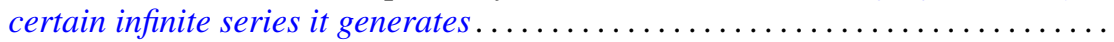

Allan L. Edelson, Conjugations on stably almost complex manifolds . ...........

Patrick John Fleury, Hollow modules and local endomorphism rings . . ..........

Jack Tilden Goodykoontz, Jr., Connectedness im kleinen and local connectedness in

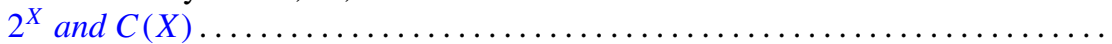

Robert Edward Jamison, II, Functional representation of algebraic intervals .......

Athanassios G. Kartsatos, Nonzero solutions to boundary value problems for

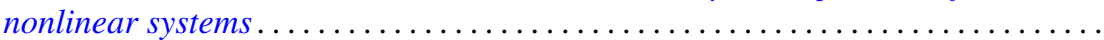

Soon-Kyu Kim, Dennis McGavran and Jingyal Pak, Torus group actions on simply

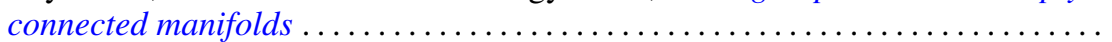

David Anthony Klarner and R. Rado, Arithmetic properties of certain recursively

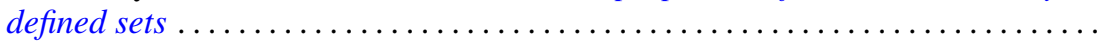

Ray Alden Kunze, On the Frobenius reciprocity theorem for square-integrable

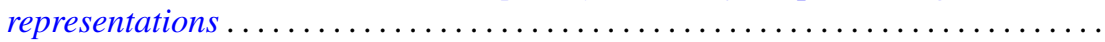

John Lagnese, Existence, uniqueness and limiting behavior of solutions of a class of differential equations in Banach space...

Teck Cheong Lim, A fixed point theorem for families on nonexpansive mappings Lewis Lum, A quasi order characterization of smooth continua

Andy R. Magid, Principal homogeneous spaces and Galois extensions . .

Charles Alan McCarthy, The norm of a certain derivation ..... . .

Louise Elizabeth Moser, On the impossibility of obtaining $S^{2} \times S^{1}$ by elementary surgery along a knot. .

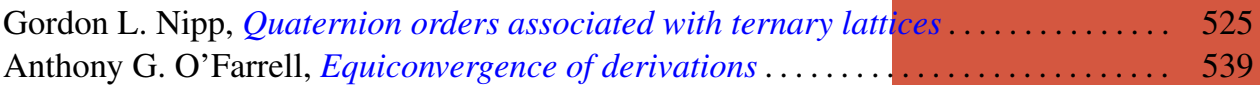

Dorte Olesen, Derivations of $A W^{*}$-algebras are inner . . . . . . . . . . . . . . . 555

Dorte Olesen and Gert Kjærgaard Pedersen, Derivations of $C^{*}$-algebras have

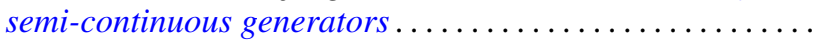

Duane O’Neill, On conjugation cobordism.

Chull Park and S. R. Paranjape, Probabilities of Wiener paths crossing differentiable

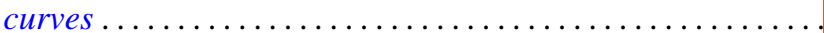

Edward Ralph Rozema, Almost Chebyshev subspaces of $L^{1}(\mu$;

Lesley Millman Sibner and Robert Jules Sibner, A note on the Atiyah-Bott fixed

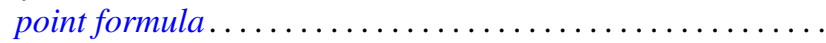

Betty Salzberg Stark, Irreducible subgroups of orthogonal groups generated by

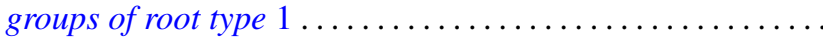

N. Stavrakas, A note on starshaped sets, $(k)$-extreme points and the half ray

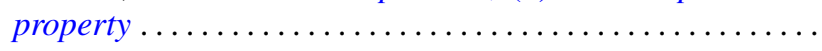

Carl E. Swenson, Direct sum subset decompositions of $Z \ldots \ldots$ 